\title{
The effect of glycemic control on executive functions in elderly patients with type 2 diabetes mellitus.
}

\author{
Hend F Mahmoud*, Marwa A Abdel gawad \\ Faculty of Medicine, Department of Geriatrics and Gerontology, Ain Shams University, Cairo, Egypt
}

\begin{abstract}
Introduction: DM is a major health problem. It is well known that DM has a deleterious effect on vascular, retinal, renal, and peripheral nerve functions. A growing evidence suggests that elderly with type 2 DM have impairments in executive functions. The aim of the study was to detect the effect of glycemic control, duration of DM and its complications on executive functions.

Methods: A cross-sectional observational study was conducted in a sample comprised of two hundred elderly patients with type 2 diabetes. Glycemic control, duration of diabetes, complications of diabetes and executive functions were assessed

Results: There was a significant correlation between glycemic control and neuropsychological tests assess executive functions except for the clock drawing test. Also, there was a significant correlation between duration of diabetes and the number of diabetes complications and executive functions tests.

Conclusion: Poor glycemic control is associated with worse executive functions, also longer duration of diabetes and increasing number of diabetes complications are associated with poorer executive functions.
\end{abstract}

Keywords: Type 2 DM; Duration of diabetes; HbA1c; Executive functions.

Accepted on November 272018

\section{Introduction}

$\mathrm{DM}$ is a major health problem. WHO stated that the number of people with diabetes has increased from 108 million in 1980 to 422 million in 2014 and proposes that diabetes will be the seventh leading cause of death in 2030 [1]. The incidence of type $2 \mathrm{DM}$ is among the most common chronic diseases continue to rise because of the aging population, urbanization, and increasing prevalence of physical inactivity and obesity [2].

It is well known that DM has a deleterious effect on vascular, renal, retinal, vascular and peripheral nerve functions. In addition to these well-Known complications type 2 DM has been identified as a significant risk factor for cognitive impairment and dementia [3]. Previous studies results showed that older adults with diabetes have increased risk of dementia, and that poor glycemic control leads to cognitive dysfunction $[4,5]$

Executive function is a domain of cognition, it involves a broad set of cognitive abilities such as working memory, attention, organization and inhibition of inappropriate behaviour that are necessary for performing complex, goal-directed activities. These functions are often related to the frontal lobe, because they are dependent on the frontal cortex and its networks in other cerebral and subcortical areas [6].

Recently it is suggested that elderly with type 2 DM have executive dysfunction so higher attention is being directed to the connection between diabetes and executive functioning domain of cognition now. Recent data suggest that executive dysfunction is considered a risk factor for poor blood sugar control [7]. Executive dysfunction is associated with poor self- care, including low autonomy, inability to make decisions, low independence in instrumental activities of daily living, poor adherence to medication and also resistance to care [8].

Physiological data enforce the likelihood of executive functions affection in older adults with type $2 \mathrm{DM}$, due to the neuroanatomical changes resulting from impaired glycemic control, insulin resistance and vascular disease [9]. Neuroanatomical changes in diabetic patients include cortical and subcortical brain atrophy and significantly deeper white matter lesions and infarcts than persons without DM [10]. Impaired glycemic control has long been suspected to contribute to the development of cognitive impairment in diabetic patients. [9] Animal models have indicated that hyperglycemia may induce the formation of reactive oxygen species and advanced glycation end products, the activation of protein kinase $\mathrm{C}$ and polyol pathways, increased glucose shunting in the hexosamine pathway, and affection of neurotransmitter functions [9]. Vascular dysfunction may contribute to the development of executive disturbance. There is a speculation that the combination of reduced cerebral flow and activation of Thromboxane A2 receptor may disrupt cerebral vasodilatation. The resulting ischemia may be exacerbated by hyperglycemia providing an environment in which damaging agents as lactate or glutamate can accumulate and cause neural injury [9]. Recent evidence suggests that insulin resistance may enhance the development or inhibit the degradation of the $\beta$ amyloid plaques characteristic for Alzheimer disease [9]. 


\section{Materials and Methods}

A cross-sectional observational study was conducted to investigate the effect of glycemic control, duration of DM and its complications on executive functions. Participants were 200 elderly patients with past history of type 2 diabetes mellitus aged 60 years and above both males and females recruited from geriatric outpatient clinic and geriatric department in Ain Shams University Hospital. Exclusion criteria included patients under 60 years, illiterate patients, patients with disturbed level of consciousness due to any medical condition or drug effect, patients with delirium, patients with other comorbidities affecting executive functions (e.g. cerebrovascular stroke, chronic kidney disease) and those who refused to participate. Data collection included detailed medical history with duration of diabetes and presence of complications, clinical examination, mini-mental state examination, geriatric depression scale activities of daily living (ADLs), instrumental activities of daily living (IADLs), all patients underwent executive functions assessment using EXIT 25, animal verbal fluency, digit span forward, digit span backward and clock drawing test [11-18]. Glycemic control was assessed by measuring HbAlc, FBS and 2HPP through a venous blood sample for each patient. Informed consent was obtained from all participants.

\section{Data analysis}

Analysis of data was performed by the $16^{\text {th }}$ version of Statistical Package for Social Science (SPSS). Description of quantitative data was done in the form of Mean (M), Standard Deviation (SD) and range. Frequency and percentage were done for qualitative variables. Spearman's correlation coefficient was used for non-parametric correlations. Significance level measured according to $\mathrm{P}$ value (probability), $\mathrm{P}>0.05$ for insignificant, $\mathrm{P}<0.05$ for significant and $\mathrm{P}<0.01$ for highly significant.

\section{Results}

The study included 200 participants $56 \%$ women and $44 \%$ men. Their age ranged from 60 to 77 years and the mean age was $65.5 \pm 4.8$ years. $77 \%$ of the participants were not working and $23 \%$ were working. $925 \%$ of our patients were living alone but the rest $75 \%$ living with others. Most of them $65 \%$ were educated for 5 years and $65 \%$ of them were non-smokers (Table 1).

There was a significant positive correlation between $\mathrm{HbA1c}$, FBS and 2HPP with EXIT 25 and a significant negative correlation with Animal verbal fluency, Digit span forward and Digit span backward. Regarding the Clock drawing test there was a significant negative correlation with $\mathrm{HbA1c}$ and a nonsignificant negative correlation with FBS and 2 hr PP (Table 2).

Table 1. Demographic characteristics of cases.

\begin{tabular}{|c|c|c|}
\hline \multirow{2}{*}{ Age (Yr; Mean \pm SD) } & Mean \pm SD & Range \\
\hline & $65.5 \pm 4.8$ & $60-77$ \\
\hline \multicolumn{3}{|l|}{ Gender } \\
\hline & $\mathrm{N}$ & $\%$ \\
\hline Male & 88 & $44 \%$ \\
\hline Female & 112 & $56 \%$ \\
\hline \multicolumn{3}{|l|}{ Occupation } \\
\hline Working & 46 & $23 \%$ \\
\hline No working & 154 & $77 \%$ \\
\hline \multicolumn{3}{|l|}{ living arrangement } \\
\hline with others & 150 & $75 \%$ \\
\hline alone & 50 & $25 \%$ \\
\hline \multicolumn{3}{|l|}{ Education } \\
\hline 5 yrs education & 112 & $56 \%$ \\
\hline $6-10$ yrs education & 66 & $33 \%$ \\
\hline$>10$ yrs education & 11 & $5.5 \%$ \\
\hline \multicolumn{3}{|l|}{ Smoking } \\
\hline No & 130 & $65 \%$ \\
\hline Yes & 70 & $35 \%$ \\
\hline
\end{tabular}

Duration of DM also had significant positive correlation with EXIT 25 and significant negative correlation with Animal verbal fluency, Digit span forward and backward and Clock drawing test (Table 2).

The patients were classified as regard diabetic complications to 3 categories (not complicated, had only one complication either "neuropathy or retinopathy or nephropathy" and multiple complications). It was found that number of diabetic complications had a significant positive correlation with EXIT 25 and significant negative correlation with Animal verbal fluency, Digit span forward and backward and Clock drawing test (Table 2).

Table 2. The correlation of HbAlc and plasma glucose level with executive functions.

\begin{tabular}{|l|l|l|l|l|l|l|}
\hline \multicolumn{2}{|l|}{ Spearman's rho correlation } & & HbA1c & FBS & 2 HPP & $\begin{array}{l}\text { duration of DM in } \\
\text { years }\end{array}$ \\
\hline \multirow{2}{*}{ EXIT 25} & Correlation Coefficient & 0.508 & 0.316 & 0.208 & 0.345 \\
\cline { 2 - 7 } & Sig. (2-tailed) & .000 & .000 & 0.003 & .000 \\
\hline
\end{tabular}


Citation: Hend F Mahmoud, Marwa A Abdel gawad. The effect of glycemic control on executive functions in elderly patients with type 2 diabetes mellitus. J Ment Health Aging 2018;2(2):56-60.

\begin{tabular}{|c|c|c|c|c|c|c|}
\hline \multirow{2}{*}{$\begin{array}{l}\text { Animal verbal fluency (no. of } \\
\text { animals) }\end{array}$} & Correlation Coefficient & -0.505 & -0.289 & -0.210 & -0.356 & -0.397 \\
\hline & Sig. (2-tailed) & .000 & .000 & 0.003 & .000 & .000 \\
\hline \multirow{2}{*}{ Digit span forward } & Correlation Coefficient & -0.369 & -0.517 & -0.429 & -0.235 & -0.144 \\
\hline & Sig. (2-tailed) & .000 & .000 & .000 & 0.001 & 0.042 \\
\hline \multirow{2}{*}{ Digit span backward } & Correlation Coefficient & -0.420 & -0.271 & -0.198 & -0.409 & -0.346 \\
\hline & Sig. (2-tailed) & .000 & .000 & 0.005 & .000 & .000 \\
\hline \multirow{2}{*}{ Clock drawing test score } & Correlation Coefficient & -0.447 & -0.108 & -0.047 & -0.400 & -0.439 \\
\hline & Sig. (2-tailed) & .000 & 0.128 & 0.513 & .000 & .000 \\
\hline
\end{tabular}

\section{Discussion}

Glycemic control is an important element of diabetes management. Many older adults can't achieve or maintain glycemic control [19]. Impaired cognition may contribute to this. Effective control of blood glucose level involves a series of complex goal-directed behaviours including proper nutrition, regular exercise, medication management and selfmonitoring of blood glucose [20]. A patient's cognitive ability to execute these behaviours is essential for diabetes selfmanagement.

Previous studies found that cognitive capacity affects individuals' ability to achieve glycemic control, and poor glycemic control results in impairment of cognitive function in adults with diabetes, this suggests the possibility of presence of bidirectional association [7].

Impaired performance on a variety of executive tasks has been reported in older adults with type $2 \mathrm{DM}$ and a significantly increased risk of executive decline has been observed on longitudinal investigations of type $2 \mathrm{DM}$ and cognition [21]

Executive functions are complex cognitive processes involved in control and regulation of simple cognitive actions as well as goal directed and future oriented behaviours. It involves multiple domains as attention, working memory, cognitive flexibility decision making, judgment, and behavioural regulation [22].

The current study aimed at investigating the effect of glycemic control, duration of DM and presence of diabetes complications on executive functions in elderly patients with type 2 DM. It involved 200 elderly patients with type 2 DM both males and females.

Assessment of different executive functions domains was done by using 5 neurophysiological tests, these tests are:

EXIT 25: assesses planning, initiation, and regulation of complex goal directed behavior, Digit span forward and backwards are used to assess working memory, working memory is the ability to hold information for a brief period and to manipulate it. Animal verbal fluency test was used to assess semantic memory, the clock drawing test used to assess planning, organization, and visuospatial skill [15-18].

The current study provided evidence for the presence of executive dysfunction in elderly patients with type 2 diabetes mellitus. These findings are consistent with the results of the study done by Qiu et al. [23], who performed a cross-sectional study that showed that diabetic patients' speed in processing and executive functions were markedly lower than others.

The results of this study showed a significant positive correlation between HbA1c, FBS and 2HPP and EXIT 25. It also showed a significant negative correlation between $\mathrm{HbAlc}$, FBS and 2HPP and digit span forward, digit span backwards and animal verbal fluency tests. The clock drawing test had a non-significant negative correlation with $\mathrm{HbA1c}$, FBS and 2HPP.

Several cross sectional studies found that uncontrolled diabetes is associated with poor cognitive functions, In a study done to evaluate the relationship between the degree of hyperglycemia which was assessed by HbAlc and FBS and cognitive status which was assessed by the Digit Symbol Substitution Test (DSST), Mini Mental Status Examination [MMSE], Rey Auditory Verbal Learning Test, and Stroop Test it found that Higher A1C levels are associated with lower cognitive function in individuals with diabetes [24].

Another cross-sectional study performed by Grober et al. [25] to investigate the relation between glycemic control, memory impairment and executive dysfunction in elderly patients with diabetes found that Memory impairment and executive function affection were associated with inadequately controlled diabetes. Executive function was assessed by an ordinal composite score derived from animal fluency and months backward.

These results disagree with the results of Action to Control Cardiovascular Risk in Diabetes (ACCORD) Study which enrolled 2977 patients (mean age $=62.5$ years; $S D=5 \cdot 8$ ) with type 2 diabetes divided into 2 groups. The first group receives intensive glycaemic control targeting $\mathrm{HbA} 1 \mathrm{c}$ to less than $6.0 \%$ and the second group receives a standard strategy targeting $\mathrm{HbA} 1 \mathrm{c}$ to $7.0-7.9 \%$. Cognitive assessment was done using the Digit Symbol Substitution Test (DSST) score, at baseline and at 20 and 40 months. The results revealed that there was no significant difference between 2 groups in means 40-month DSST score [26]. The discrepancy in the results between both studies may be attributed to narrow $\mathrm{HbAlc}$ range in the ACCORD study as it compared cognitive functions in intensive (HbAlc less than 6.0\%) versus standard glycemic control (HbA1c 7.0-7.9\%) while in the current study 
correlation was done between executive function and glycemic control with wider HbAlc range (5.8-11.5\%). Besides, Different tests to assess cognitive functions have been used in each study. In the ACCORD study the Digit symbol Substitution Test is used while in this study 5 neuropsychological tests have been used to evaluate different domains of executive functions. These factors can explain such variation in the results

Long duration of DM may be associated with greater cerebral macro vascular disease, clinical and subclinical cerebral infarctions that may affect cognitive function [27]

In the current study there was a statistically significant positive correlation between diabetes duration and EXIT 25 test and a statistically significant negative correlation between diabetes duration and other neuropsychological tests used to assess executive functions. These results are in accordance with those of a study done to evaluate the relationship between T2D and cognitive impairment, and the subjects with diabetes had lower MMSE score than those without diabetes. Also, a statistically significant correlation between the duration of the disease and cognitive dysfunction was observed [28]

This study also showed that the number of diabetic complications had a statistically significant correlation with all neuropsychological tests used to assess executive functions.

According to some studies, the vascular complications of diabetes such as retinopathy are the most important predictors for the cognitive decline [29]. A longitudinal study from Japan confirmed the association between diabetic retinopathy and cognitive impairment [30]. Another study showed that diabetic patients with lower limb amputation because of peripheral vascular disease had lower cognitive functioning especially in the areas of memory and executive functions [31]

\section{Conclusion}

This study assessed the effect of glycemic control on executive functions in elderly patients with type 2 diabetes. It concluded that poor glycemic control is associated with worse executive functions, also the findings suggest that longer duration of diabetes and increasing number of diabetes complications are associated with poorer executive functions.

\section{Recommendations}

- Physicians should consider follow up and control of HbA1c to avoid poor glycemic control and its complications.

- Assessment of executive functions in diabetic patients is recommended on diagnosis and periodically for early detection of executive dysfunction.

- Special concern for patients with longer duration of diabetes mellitus and continuous follow up of their executive and cognitive functions.

- Try to prevent diabetic complications via continuous follow up and good glycemic control.
- Encouragement of diabetic patients to attend educational and self-management sessions for better quality of life and better control.

- Future studies to investigate the effect of other factors such as BMI, dyslipidemia and other comorbidities on executive functions.

\section{References}

1. Mathers CD, Loncar D. Projections of global mortality and burden ofdisease from 2002 to 2030. PLoS Med. 2006;3(11):e442.

2. Wild S, Roglic G, Green A, et al. Global prevalence of diabetes: estimates for the year 2000 and projections for 2030. Diabetes care. 2004; 27:1047-1053.

3. Arvanitakis Z, Wilson RS, Bennett DA. Diabetes mellitus, dementia, and cognitive function in older persons. J Nutr Health Aging. 2006;10:287-291.

4. Xu W, Qiu C, Gatz M, et al. Mid-life and late-life diabetes in relation to the risk of dementia: A population-based twin study. Diabetes. 2009;58(1):71-77.

5. Messier C. Impact of impaired glucose tolerance and type 2 diabetes on cognitive aging. Neurobiol Aging. 2005;26:2630.

6. Stuss DT, Levine B. Adult clinical neuropsychology: lessons from studies of the frontal lobes. Annu Rev Psychol. 2002;53:401-433.

7. De Wet H, Levitt N, Tipping B. Executive cognitive impairment detected by simple bedside testing is associated with poor glycaemic control in type 2 diabetes. S Afr Med J. 2007;97(11):1074-1076.

8. Schillerstrom JE, Horton MS, Royall DR. The impact of medical illness on executive function. Psychosomatics. 2005;46(6):508-516.

9. Kodli CT, Seaquist ER. Cognitive dysfunction and diabetes mellitus. Endocr Rev. 2008;29:494-511.

10. Manschot SM, Brands AM, van der Grond J, et al. Brain magnetic resonance imaging correlates of impaired cognition in patients with type 2 diabetes. Diabetes. 2006;55:1106:1113.

11. Folstein MF, Folstein SE, McHug PR. Mini-mental state. A practical method for grading the cognitive state of patients for clinicians. J Psychiatry Res. 1975;12(3):189-98.

12. Sheikh JA, Yesavage JA. Recent finding and development of a shorter version. In Brinn TL[Ed]. Clinical gerontology: A guide to assessment and intervention, New York, Hawarth Press. 1986.

13. Katz S, Down TD, Cash HR. Progress in the development of the index of ADL. J Gerontol. 1970;10:20-30.

14. Lawton MP, Brody EM. Assessment of older people: Selfmaintaining and instrumental activities of daily living. J Gerontol. 1965;9:179-186.

15. Royall DR, Mahurin RK, Gray KF. Bedside assessment of executive cognitive impairment: The executive Interview. J Am Geriatr Soc. 1992;40:1221-1226.

16. Morris JC, Heyman A., Mohs RC, et al. The consortium to establish a registry for Alzheimer's disease. Part I: clinical 
Citation: Hend F Mahmoud, Marwa A Abdel gawad. The effect of glycemic control on executive functions in elderly patients with type 2 diabetes mellitus. J Ment Health Aging 2018;2(2):56-60.

and neuropsychological assessment of Alzheimer's disease. Neurology. 1989;39:1159-1165.

17. Wechsler D. Wechsler Adult intelligence scale. 3rd edition (WAIS-3R) San Antonio, TX: Harcourt Assessment. 1997.

18. Watson YI, Arfken CL, Birge SJ. Clock completion: An objective screening test for dementia. J Am Geriatr Soc. 1993;41:1235-1240.

19. Quandt SA, Bell RA, Snively BM, et al. Ethnic disparities in glycemic control among rural older adults with type 2 diabetes. Ethn Dis. 2005;15(4):656-663.

20. Glasgow RE, Fisher L, Skaff M, et al. Problem solving and diabetes self-management: investigation in a large, multiracial sample. Diabetes Care. 2007;30(1):33-37.

21. Van den Berg E, Reijmer YD, de Bresser J, et al. A 4 year follow up study of cognitive functioning in patients with type 2 diabetes mellitus. Diabetologia. 2010;53:58-65,

22. Spreen O, Strauss E. A compendium of neuropsychological tests: administration, norms and commentary. New York: oxford university. 1998.

23. Qiu C, Sigurdsson S, Zhang Q, et al. Diabetes, markers of brain pathology and cognitive function: the Age, Gene/ Environment Susceptibility. Ann Neurol. 2014;75(10): 138-46.

24. Cukierman-Yaffe T, Gerstein HC, Williamson JD, et al. Relationship between baseline glycemic control and cognitive function in individuals with type 2 diabetes and other cardiovascular risk factors: The action to control cardiovascular risk in diabetes- memory in diabetes (ACCORD-MIND) trial. Diabetes care. 2009;32:221-6.

25. Grober E, Hall CB, Hahn SR, et al. Memory impairment and executive dysfunction are associated with inadequately controlled diabetes in older adults. J primary care community health. 2011;2:229-33.

26. Launer LJ, Miller ME, Williamson JD, et al. E $\square$ ects of intensive glucose lowering on brain structure and function in people with type 2 diabetes [ACCORD MIND]: A randomised open-label substudy. Lancet. 2011;10:969-77.

27. Vermeer SE, Den Hejjer T, Koudstaal PJ, et al. Scan study, incidence and risk factors of silent brain infarcts in the population-based Rotterdam scan study. Stroke. 2003;34(2):392- 396.

28. Ebady SA, Arami MA, Shafigh MH. Investigation on the relationship between diabetes mellitus type 2 and cognitive impairment. Diabetes Res Clin Pract. 2008;82:305-309.

29. Patton N, Aslam T, Macgillivray T, et al. Retinal vascular image analysis as a potential screening tool for cerebrovascular disease: A rationale based on homology between cerebral and retinal microvasculatures. J Anat. 2005; 206:319-348.

30. Kadoi Y, Saito S, Fujita N, et al. Risk factors for cognitive dysfunction after coronary artery bypass graft surgery in patients with type 2 diabetes. J Thorac Cardiovasc Surg. 2005;129:576-583.

31. Coffey L, O'Keeffe F, Gallagher P, et al. Cognitive functioning in persons with lower limb amputations: A review. Disabil. Rehabil. 2012; 34:1950-1964.

\section{*Corresponds to:}

Hend Fawzy Mahmoud

Department of Geriatrics and Gerontology,

Faculty of Medicine,

Ain Shams University, Cairo,

Egypt.

Tel: 02-22848272

E-mail: hm.hend@yahoo.com 